\title{
Screening and analysis of PoAkirin1 and two related genes in response to immunological stimulants in the Japanese flounder (Paralichthys olivaceus)
}

\author{
Chang-Geng Yang ${ }^{1,2}$, Xian-Li Wang ${ }^{3}$, Bo Zhang ${ }^{4}$, Bing Sun ${ }^{1}$, Shan-Shan Liư ${ }^{1}$ and Song-Lin Chen ${ }^{1 *}$
}

\begin{abstract}
A member of the NF-kB signaling pathway, PoAkirin1, was cloned from a full-length CDNA library of Japanese flounder (Paralichthys olivaceus). The full-length cDNA comprises a 5'UTR of 202 bp, an open reading frame of 564 bp encoding a 187-amino-acid polypeptide and a 521-bp 3'UTR with a poly (A) tail. The putative protein has a predicted molecular mass of $21 \mathrm{kDa}$ and an isoelectric point (pl) of 9.22. Amino acid sequence alignments showed that PoAkirin 1 was 99\% identical to the Scophthalmus maximus Akirin protein (ADK27484). Yeast two-hybrid assays identified two proteins that interact with PoAkirin1: PoHEPN and PoC1q. The CDNA sequences of PoHEPN and PoC1q are $672 \mathrm{bp}$ and $528 \mathrm{bp}$, respectively. Real-time quantitative reverse-transcriptase polymerase chain reaction analysis showed that bacteria could induce the expressions of PoAkirin1, PoHEPN and PoC1q. However, the responses of PoHEPN and PoClq to the bacterial challenge were slower than that of PoAkirin1. To further study the function of PoAkirin1, recombinant PoAkirin1 and PoHEPN were expressed in Escherichia coli and would be used to verify the PoAkirin1-PoHEPN binding activity. These results identified two proteins that potentially interact with PoAkirin 1 and that bacteria could induce their expression.
\end{abstract}

Keywords: Akirin, Japanese flounder, NF-kB, Yeast two-hybrid assay, Immunity, HEPN, C1q

\section{Background}

Biological processes are primarily performed and controlled by proteins. Therefore, clarifying the biological functions of proteins and their biological response mechanisms at the cellular level has become the main objective of proteomics research. Protein-protein interactions play a crucial role in various biological functions, including the formation of polymer structure, cell signal transduction, gene regulation, and metabolic pathways. In the post-genome sequencing era, protein interaction bridges the gap between prediction of the relationship between the proteins and the annotation of important genes. Thus, comprehensive analysis of protein-protein interactions is crucial for the full understanding of proteomics [1]. Studies of protein-protein interactions not

\footnotetext{
* Correspondence: chens|@ysfri.ac.cn

${ }^{1}$ Yellow Sea Fisheries Research Institute, Chinese Academy of Fisheries

Sciences, Qingdao 266071, China

Full list of author information is available at the end of the article
}

only can reveal the protein function on the molecular level but also are critical for understanding growth, development, differentiation and apoptosis and other crucial life activities, such studies also provide an important theoretical basis for disease mechanisms, disease treatment, disease prevention and drug development. The yeast two-hybrid system is a simple, but powerful, tool for detecting interactions between proteins and has been widely applied in many research areas. Recently, the yeast two-hybrid system has been used to study the large-scale interaction group in viruses, bacteria, Drosophila, and Caenorhabditis elegans [2-7].

Nuclear factor $\kappa B(N F-\kappa B)$ is a nuclear transcription factor that plays a key role in the regulation of apoptosis, viral replication, cancer, inflammation and the regulation of the expression of other related genes. In particular, NF- $\mathrm{kB}$ can be activated by a variety of stimulatory factors, including cytokines, 
lymphokines, UV, pharmaceutical preparations, and growth and stress factors. Such activation of NF- $k B$ is part of the stress response. Although many members in the NF- $\mathrm{KB}$ signaling pathway have been identified in the past 20 years, a highly conserved protein Akirin, a member in the NF- $\mathrm{kB}$ signaling pathway, was recently identified in a study of the immune defense system at 2008 [8]. This 20-25-kDa protein participates in the regulation of gene expression in

Table 1 Oligonucleotide primers used in this study

\begin{tabular}{|c|c|c|}
\hline Name & Sequence & Purpose used \\
\hline AKI-F-S1 & ATGGCCTGCGGAGCGACGTT & ORF amplification \\
\hline AKI-F-A1 & TCAGGAGACATAACTAGCAGGCCG & ORF amplification \\
\hline$\beta$-actin-s1 & GCTGTGCTGTCCCTGTA & RT-PCR \\
\hline$\beta$-actin-a1 & GAGTAGCCACGCTCTGTC & RT-PCR \\
\hline GAP-S1 & CAACGGCGACACTCACTCCTC & RT-PCR \\
\hline GAP-A1 & TCGCAGACACGGTTGCTGTAG & RT-PCR \\
\hline TUBA-S1 & TGACATCACAAACGCCTGCTTC & RT-PCR \\
\hline TUBA-A1 & GCACCACATCTCCACGGTACAG & RT-PCR \\
\hline AKI-R-S1 & AGGACCAGCCCTCGTTCACACT & RT-PCR \\
\hline AKI-R-A1 & TCCGTATCTTCGCATGATCTGGT & RT-PCR \\
\hline HEPN-R-S1 & TACAAGGACAATGGTGGGGG & RT-PCR \\
\hline HEPN-R-A1 & GGCAAGGGCTGAGATGGAG & RT-PCR \\
\hline C1q-R-S1 & CTCCAGAAAACGAAGCAGGC & RT-PCR \\
\hline C1q-R-A1 & TGTCGCACATCATCAAGTGAAC & RT-PCR \\
\hline AKI-F-A7 & GCGTCGACGGAGACATAACTAGCAG & $\begin{array}{c}\text { Plasmid } \\
\text { construction }\end{array}$ \\
\hline AKI-T-A1 & GCGGTACCGGAGACATAACTAGCAG & $\begin{array}{c}\text { Plasmid } \\
\text { construction }\end{array}$ \\
\hline AD-AKI-S1 & GTGAATTCATGGCCTGCGGAGCGACGTT & $\begin{array}{l}\text { Plasmid } \\
\text { construction }\end{array}$ \\
\hline AD-AKI-A1 & CAGCTCGAGTCAGGAGACATAACTAGCAG & $\begin{array}{c}\text { Plasmid } \\
\text { construction }\end{array}$ \\
\hline $\mathrm{C} 1 \mathrm{q}-\mathrm{S} 1$ & GCCGAATTCAAGGGGGCACCAGGTCTTAA & $\begin{array}{c}\text { Plasmid } \\
\text { construction }\end{array}$ \\
\hline $\mathrm{C} 1 \mathrm{q}-\mathrm{A} 1$ & CAGGTCGACTCAGGCCGTGGGGAAGACGA & $\begin{array}{l}\text { Plasmid } \\
\text { construction }\end{array}$ \\
\hline HEPN-S3 & GTGAATTCGGCACGAGGCTCAGGTGGCA & $\begin{array}{c}\text { Plasmid } \\
\text { construction }\end{array}$ \\
\hline HEPN-A2 & CAGCTCGAGAGCCTCCTCTTTGTTTGGCC & $\begin{array}{c}\text { Plasmid } \\
\text { construction }\end{array}$ \\
\hline HEPN-A3 & CAGCTCGAGGGAAGGTTGACCGTGCCTTT & $\begin{array}{l}\text { Plasmid } \\
\text { construction }\end{array}$ \\
\hline HEPN-S5 & AGTGAATTCTCTGGCAGCTCCATCTCAGC & $\begin{array}{c}\text { Plasmid } \\
\text { construction }\end{array}$ \\
\hline HEPN-A4 & CAGCTCGAGCTATTTCACATATGCCTCAAC & $\begin{array}{c}\text { Plasmid } \\
\text { construction }\end{array}$ \\
\hline HEPN-ET-S1 & CCGAATTCAGGCTCAGGTGGCATCCT & $\begin{array}{l}\text { Plasmid } \\
\text { construction }\end{array}$ \\
\hline HEPN-ET-A1 & GTGCTCGAGCTATTTCACATATGCCTCA & $\begin{array}{c}\text { Plasmid } \\
\text { construction }\end{array}$ \\
\hline
\end{tabular}

many physiological processes, including the insect and mammalian innate immune response [8], cancer, insect reproduction and arthropods growth $[9,10]$. Knockout of the Akirin gene led to embryonic lethality of mice and caused death or reduced growth in Drosophila, ticks, and nematodes. Consequently, Akirin is considered important in animal development [11]. Akirin cannot directly combine with DNA, but interacts with the promoter or assisting factors that inhibit the transcription of genes encoding such proteins as the 14-3-3 protein and the helix-loop-helix transcription factor, Twist [12]. Research on fish Akirin has been limited to the analysis of gene structure and function in several species [13-15]. Furthermore, Akirin's interaction mechanism requires further investigation.

The Japanese flounder (Paralichthys olivaceus) is economically important and is widely cultured in Europe and China. However, flounder diseases have a serious impact on the aquaculture industry. Recently, to explore the molecular mechanisms of disease resistance and host-pathogen interactions in this species, Nam's team $[16,17]$ constructed a cDNA library from an immune stimulated Japanese flounder. In addition, many immune-related genes including those encoding STAT, Nramp (natural resistance associated macrophage protein), MHCIIA and IIB (major histocompatibility complex class II) have also been investigated [18-20].

As an important protein required for NF- $\mathrm{kB}-$ dependent gene regulation in the immune response, little is known about Akirin's function, interacting proteins, and regulation mechanism. In this study, we

Table 2 Primer names and restriction sites used to construct the expression vectors

\begin{tabular}{ccc}
\hline Name & Restriction site & Vector \\
\hline AKI-F-A7 & Sall & pGBKT7-PoAkirin1 \\
AKI-T-A1 & Kpnl & \\
AD-AKI-S1 & ECoRl & pGADT7-PoAkirin1 \\
AD-AKI-A1 & Xhol & \\
C1q-S1 & EcoRl & pGBKT7- PoC1q \\
C1q-A1 & Sall & \\
HEPN-S3 & EcoRl & pGADT7- PoHEPN-M1 \\
HEPN-A2 & Xhol & \\
HEPN-S3 & ECoRl & \\
HEPN-A3 & Xhol & \\
HEPN-S5 & ECoRl & \\
HEPN-A4 & Xhol & pGADT7- PoHEPN-M3 \\
HEPN-ET-S1 & EcoRl & pET30a-PoHEPN \\
HEPN-ET-A1 & Xhol & \\
\hline
\end{tabular}




\begin{tabular}{|l} 
PoHEPN \\
pohepn1 \\
Figure 1 Sketch map of the PoHEPN deleted segment. PoHEPN: normal PoHEPN; pohepn 1: deletion of 96-223aa; pohepn2: deletion of 146-223aa; \\
pohepn3: deleted 1-146aa.
\end{tabular}

screened for Akirin interacting proteins and analyzed the interaction mechanism using the yeast twohybrid system and a cDNA library of flounder immune tissue. Two possible interacting proteins were identified: PoHEPN (a higher eukaryotes and prokaryotes nucleotide-binding domain (HEPN) protein) and PoC1q (complement component $\mathrm{C} 1$, q subunit). The expression profiles of PoAkirin1, PoHEPN and PoC1q were also analyzed by a bacterial challenge test. This study increases our understanding of the Akirin family, and provides a theory of flounder immunity and disease resistance mechanisms.

\section{Methods}

Ethics statement

The Yellow Sea Fisheries Research Institute's animal care and use committee waived the need for ethical approval, as this is not required in China.

\section{Experimental animals}

Japanese flounders with an average weight of $200 \mathrm{~g}$ were obtained from Haiyang Fisheries Company in Yantai and raised in a breeding tank with seawater $\left(20^{\circ} \mathrm{C}\right)$. For cloning and tissue expression analysis, RNA was extracted from 13 tissues (brain, gill, skin, muscle, fin, heart, liver, 
spleen, eye, pituitary, kidney, head kidney, and intestine) from three individuals. For the bacterial challenge experiment, RNA was extracted from three tissues (liver, spleen, and kidney) from three individuals.

\section{Bacterial challenge}

The bacterium Vibrio anguillarum, which is pathogenic in Japanese flounders, was cultured at $28^{\circ} \mathrm{C}$ to midlogarithmic growth on $2216 \mathrm{E}$ medium, centrifuged to

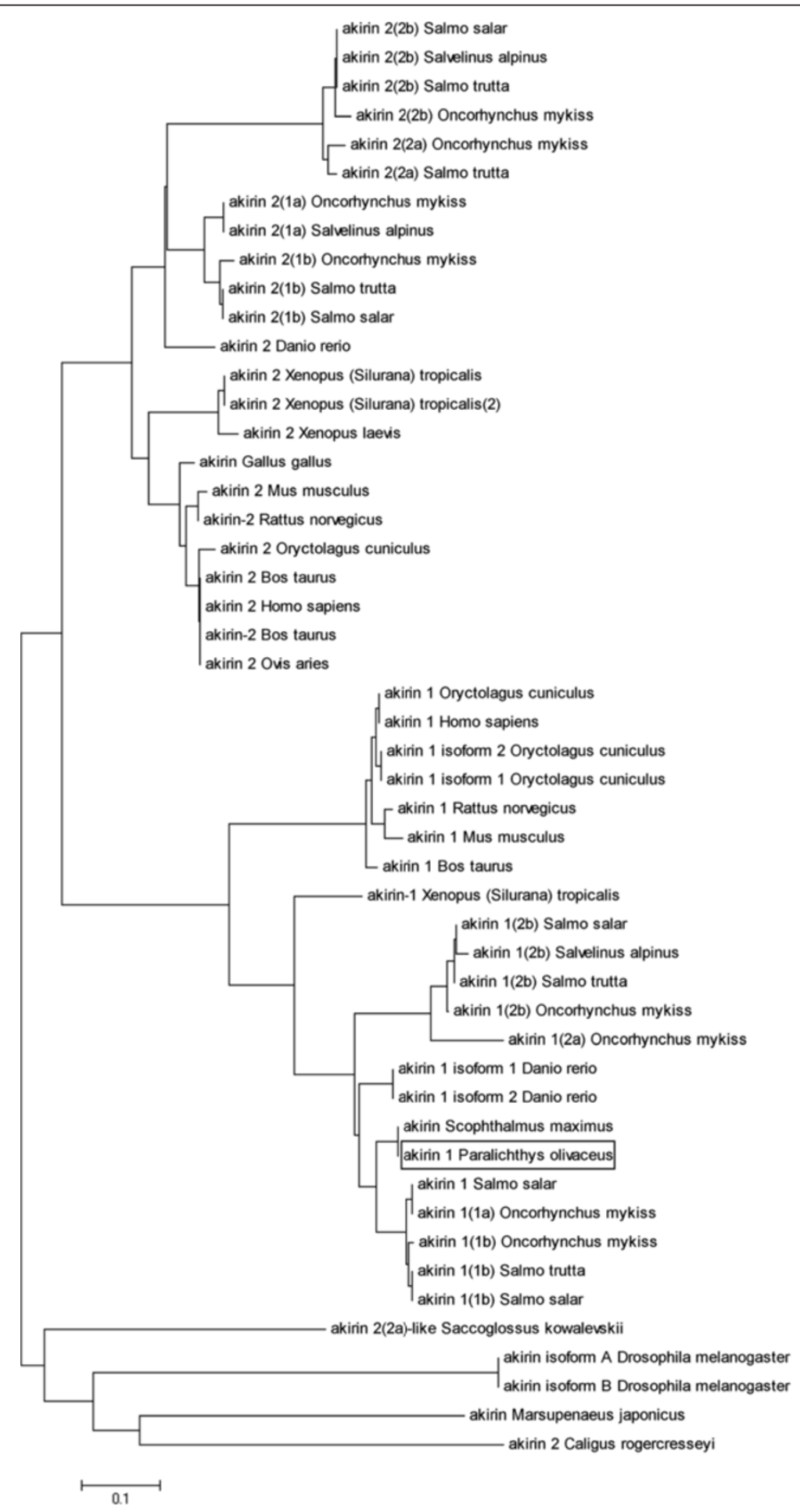

Figure 3 Phylogenetic tree, constructed by the neighbor-joining algorithm using ClustalW Multiple Alignment, showing the relationship between Akirin proteins. The PoAkirin1 protein is boxed. 
collect the bacteria and suspended in $0.9 \%$ saline $[21,22]$. A cell counter was used to measure the number of bacteria in the suspension. A final concentration of $7 \times$ $10^{6} \mathrm{cfu}$ of $V$. anguillarum was used for each injection, and $0.9 \%$ saline was used as the negative control. At $6 \mathrm{~h}, 12 \mathrm{~h}$, $24 \mathrm{~h}, 48 \mathrm{~h}, 72 \mathrm{~h}$ and $96 \mathrm{~h}$ post-injection, three individuals

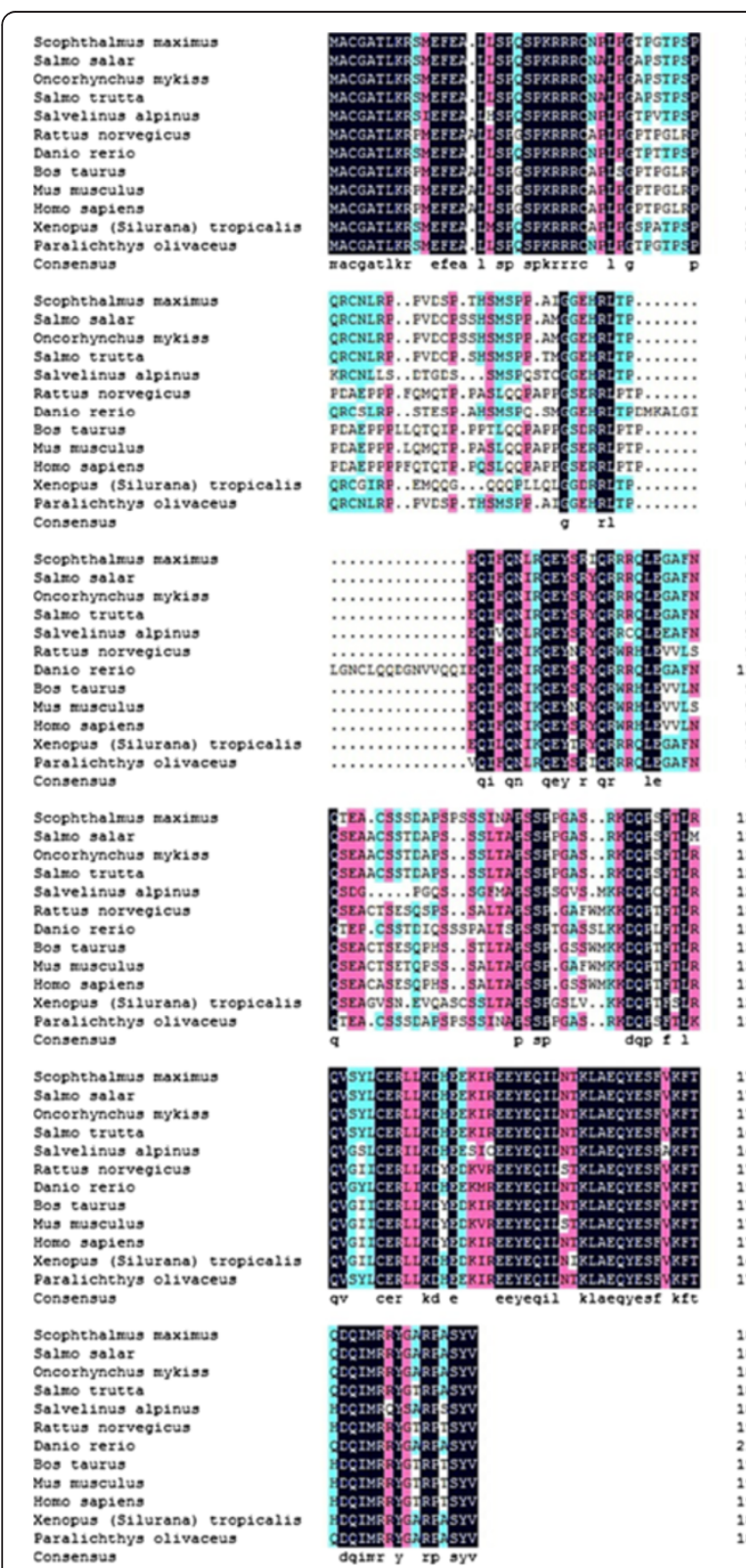

Figure 4 Alignment of amino acid sequences of PoAkirin 1 and other Akirin1 proteins. Identical residues are shown in white letters with a black background. Sequence accession nos: Scophthalmus maximus, ADK27484.1; Salmo salar, NP_001161992; Oncorhynchus mykiss, ACV49717; Salmo trutta, ACV49710; Salvelinus alpines, ACV49696; Danio rerio, NP_001025225; Rattus norvegicus, NP_001107272; Bos taurus, DAA31047; Mus musculus, AAH03291; Homo sapiens, AAl19746; Xenopus (Silurana) tropicalis, NP_001016080; Oryctolagus cuniculus, XP_002708554. from each time point were sacrificed and tissues were used for RNA extraction. For the negative control, tissues were taken $12 \mathrm{~h}$ after the saline injection.

\section{RNA extraction}

Total RNA was isolated from $500 \mathrm{mg}$ powdered fish tissues by homogenization in $5 \mathrm{~mL}$ TRIzol (Invitrogen), held at room temperature for $5 \mathrm{~min}$. An aliquot of chloroform $(1 \mathrm{~mL})$ was added to each extract, and the resulting mixture was centrifuged (10 min, 13,000 g). The aqueous layer was transferred to a clean tube, and the RNA firstly precipitated by the addition of $3 \mathrm{~mL}$ isopropanol, and then pelleted by centrifugation (15 min, $13,000 \mathrm{~g})$. The RNA pellet was washed twice with $75 \%$ ethanol and re-suspended in diethylpyrocarbonate-treated water. After DNA removal (Turbo DNA-free kit, Ambion), RNA integrity was detected using agarose gel electrophoresis, and the concentration of RNA was quantified spectrophotometrically.

\section{Cloning of PoAkirin 1}

Based on the turbot Akirin1 gene sequence, primers (AKI-R-S1 and AKI-R-A1) were designed to amplify a conservative fragment. The $5^{\prime}$ and $3^{\prime}$ fragments of PoAkirin1 gene were amplified from the flounder fulllength cDNA Library (a mix of liver, spleen and kidney).

\section{Sequence analysis}

Translation was performed using DNASTAR software. The conserved domain analysis and BLAST analysis

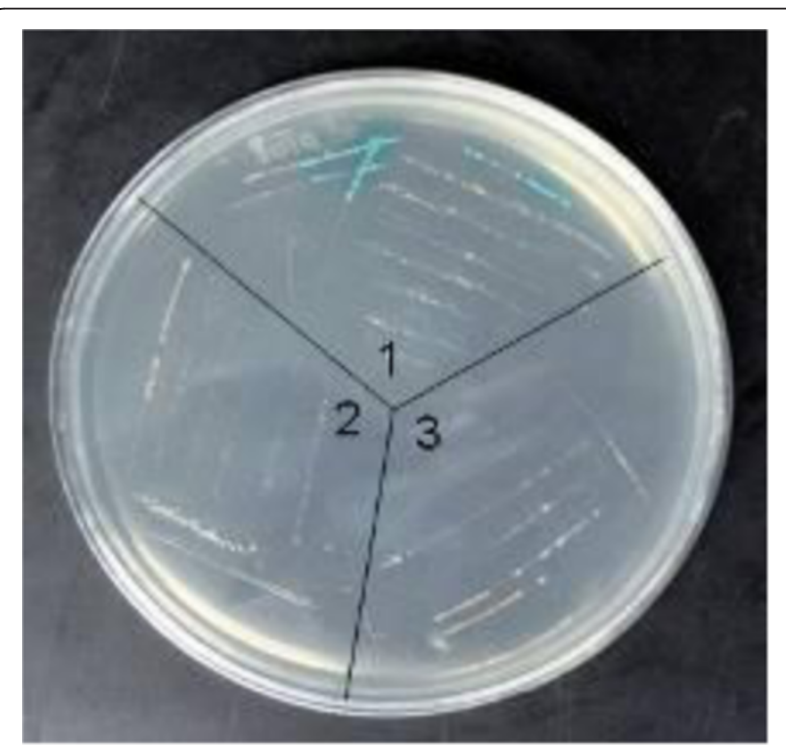

Figure 5 Transcription activation of PoAkirin1. The PoAkirin 1 gene was fused to the GAL4 DNA binding domain (GAL4-DB) in the vector pGBKT7. The positive control plasmid was pGBKT7-53. The negative control plasmid was PGBKT7-Lam. 


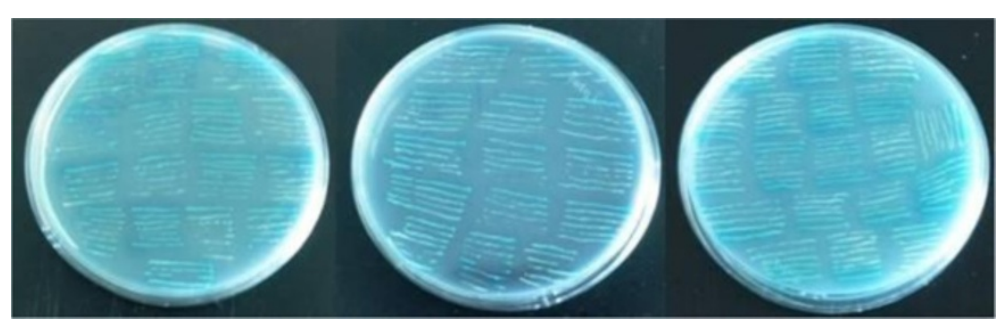

Figure 6 Forty-nine positive clones that may interact with the PoAkirin 1 from the screening library.

was performed at http://blast.ncbi.nlm.nih.gov/Blast.cgi, containing blastn, blastp and tblastp. The PSORT II server (http://psort.ims.u-tokyo.ac.jp) was used to predict the putative nuclear localization signal (NLS). The alignment of Akirins from different species was performed using the ClustalW alignment program, and the phylogenetic tree was constructed on the basis of the proportion of the amino acid differences (p-distances) determined by the neighbor-joining method [23] using MEGA 3 software [24]. The following proteins were used in the alignment: AAF50569 [Drosophila melanogaster], AAN12062 [D. melanogaster], ADK27484 [Scophthalmus maximus], BAI49701 [Marsupenaeus japonicus], ADK26453 [Gallus gallus], NP_001161992 [Salmo salar], ACV49724 [Oncorhynchus mykiss], ACV49723 [O. mykiss], ACV49722 [O. mykiss], ACV49721 [O. mykiss], ACV49720 [O. mykiss], ACV49719 [O. mykiss], ACV49718 [O. mykiss], ACV49717 [O. mykiss], ACV49716 [Salmo trutta], ACV49715 [S. trutta], ACV49714 [S. trutta], ACV49712 [S. trutta], ACV49710 [S. trutta], ACV49708 [S. salar], ACV49706 [S. salar], ACV49704 [S. salar], ACV49702 [S. salar], ACV49700 [Salvelinus alpinus], ACV49697 [S. alpinus], ACV49696 [S. alpinus], ADK39312 [Caligus rogercresseyi], NP_001107272 [Danio rerio], NP_001007187 [D. rerio], NP_988914 [Хenopus (Silurana) tropicalis], NP_001085484 [Xenopus laevis], NP_001025225 [Rattus norvegicus], DAA31047 [Bos taurus], DAA26175 [B. taurus], XP_002715780 [Oryctolagus cuniculus], XP_002714617 [O. cuniculus], XP_002708555 [O. cuniculus], XP_002708554 [O. cuniculus], XP_002736520 [Saccoglossus kowalevskii], AAH97074 [D. rerio], AAH03291 [Mus musculus], AAH61612 [X. tropicalis], CAM16479 [M. musculus],
AAI19746 [Homo sapiens], AAH05051 [H. sapiens], NP_001103557 [B. taurus], NP_001016080 [X. tropicalis], NP_001035003 [R. norvegicus], and AEO17042 [Ovis aries].

\section{Quantitative real-time RT-PCR (RT-qPCR)}

The RT-qPCR protocol adhered to the "Minimum Information for Publication of Quantitative Real-time PCR experiment guidelines [25]. The relative mRNA steadystate level was measured by RT-qPCR. The total RNA from different tissues was prepared using the TRIzol reagent. The cDNA was synthesized from each RNA sample (500 ng) using a PrimeScript ${ }^{\circ}$ RT reagent kit (Takara, China), following manufacturer's protocol. RT-qPCR was conducted on an Applied Biosystems 7500 Real-Time PCR System with SYBR $^{\circ}$ Premix Ex Taq ${ }^{\text {Ta }}$ (Takara, China). Genes encoding $\beta$-actin (for normal tissue types and infected kidney), a-tubulin (for infected spleen) and glyceraldehyde-3-phosphate dehydrogenase (for infected liver) were selected for normalization, as their expressions have been reported to be stable [26-28]. The RTqPCR primer pairs were designed to generate a product size of $150-250 \mathrm{bp}$ and a Tm of $60 \pm 1^{\circ} \mathrm{C}$. cDNA from 13 normal and infected tissues was chosen for the detection of PoAkirin1, PoHEPN and PoC1q expression, using the gene-specific primers AKI-R-S1, AKI-R-A1 (for PoAkirin1); HEPN-R-S1, HEPN-R-A1 (for PoHEPN); and C1q-R-S1, C1q-R-A1 (for PoC1q) (Table 1). The primers $\beta$-actin-s1, $\beta$-actin-a1, GAP-S1, GAP-A1, and TUBA-S1 and TUBA-A1 were used to amplify the $\beta$-actin, a-tubulin, and glyceraldehyde-3-phosphate dehydrogenase fragments, respectively (Table 1 ). PCR efficiency (E) of

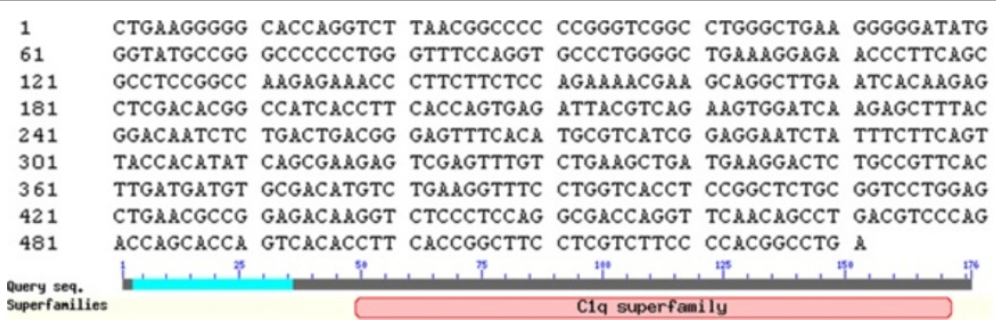

Figure 7 PoCl1q cDNA sequence and putative conserved domain. 


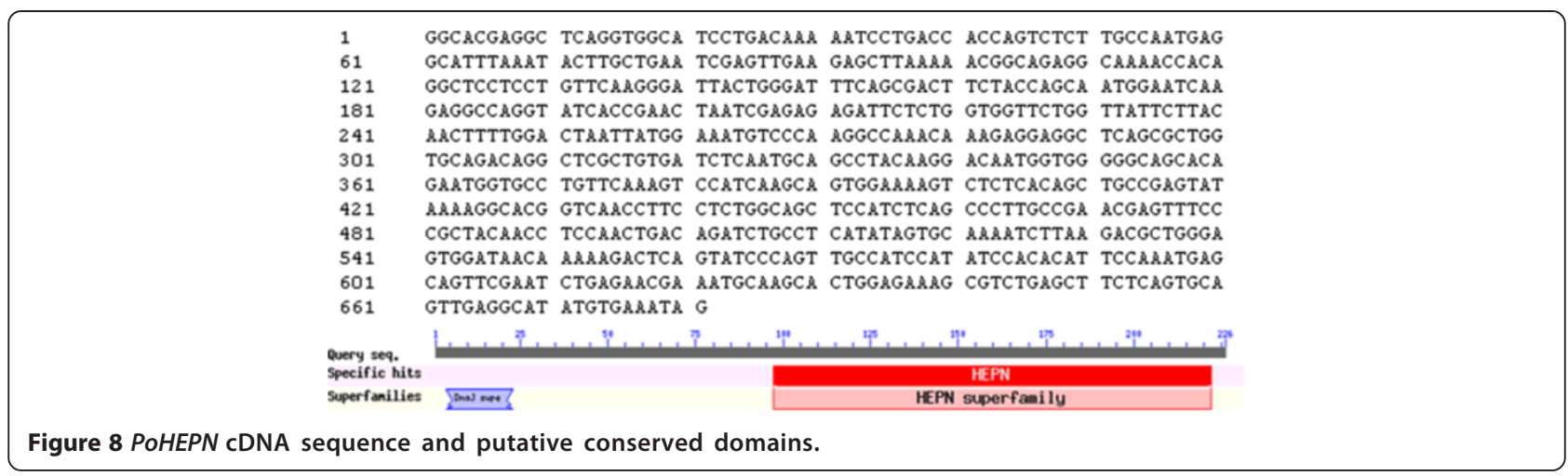

these primers were between 92 and $110 \%$ and correlation coefficient (R2) ranged from 0.991 to 0.998 .

RT-PCR was carried out with a $1 \mu \mathrm{l}$ cDNA sample, $10 \mu \mathrm{l} \mathrm{SYBR}{ }^{\circ}$ Premix Ex Taq ${ }^{\mathrm{Tm}}$, $0.4 \mu \mathrm{l}$ ROX Reference Dye II, $0.4 \mu \mathrm{l} \mathrm{PCR}$ forward/reverse primers $(10 \mu \mathrm{M})$ and $7.8 \mu \mathrm{l}$ nuclease-free water. The thermo-cycling conditions for the reaction were as follows: $95^{\circ} \mathrm{C}$ for $30 \mathrm{~s}$, followed by 40 cycles of $95^{\circ} \mathrm{C}$ for $15 \mathrm{~s}$ and $61^{\circ} \mathrm{C}$ for $34 \mathrm{~s}$. The reaction was carried out with triplicate with duplicates of each sample. Data (normalized Ct values) from the treated and control tissues templates were compared, and the 2- $\Delta \Delta C$ T method was selected for relative quantification. All data were expressed as the mean \pm S.D. and analyzed by one-way analysis of variance to determine significant differences between samples, using SPSS 16.0. Values were considered statistically significant when $\mathrm{P}<0.05$ or $\mathrm{P}<0.01$.

\section{Plasmid construction}

Using the sequences of the open reading frames (ORFs) of the PoAkirin1, PoHEPN and PoC1q genes, primers were designed to construct pGEX4T-1-PoAkirin1 (for expression in E. coli), pET30a-PoHEPN (for expression in E. coli), pGBKT7-PoAkirin1 (for Yeast two-hybrid assay), pGADT7-PoAkirin1 (for Yeast two-hybrid assay), pGBKT7-PoC1q (for Yeast two-hybrid assay), pGADT7PoHEPN-M1 (for Yeast two-hybrid assay), pGADT7PoHEPN-M2 (for Yeast two-hybrid assay), and pGADT7-PoHEPN-M3 (for Yeast two-hybrid assay) vectors. The primer sequences and restriction sites corresponding to the vectors are shown in Table 1 and Table 2. The mutation vectors pGADT7-PoHEPNM1, pGADT7-PoHEPN-M2, and pGADT7-PoHEPN-M3 mutation vectors were constructed according to the model in Figure 1.

\section{Yeast two-hybrid screening and mating assays}

The pGBKT7-PoAkirin1 plasmid and DNA plasmids for cDNA library clones were individually transformed into Saccharomyces cerevisiae strain Y2HGold and $S$. cerevisiae strain Y187, following the manufacturer's instructions in the Matchmaker ${ }^{\mathrm{rm}}$ Gold Yeast TwoHybrid System User Manual. Approximately $1 \times 10^{7}$ library clones were screened by yeast mating with selection by growth for $3-5$ days at $30^{\circ} \mathrm{C}$ on agar media lacking Leu and Trp, but with $\mathrm{X}$ - $\alpha$-galactosidase and Aureobasidin A.

\section{Recombinant protein expression in Escherichia coli}

The recombinant plasmids pGEX4T-1-PoAkirin1, pET30a-PoHEPN, pGEX4T-1, and pET30a were transformed into E. coli BL21(DE3) competent cells. After growth at $37^{\circ} \mathrm{C}, 1.0 \mathrm{mM}$ IPTG was added to the transformed cells and incubation continued for $4 \mathrm{~h}$. Sodium dodecyl sulfate polyacrylamide gel electrophoresis (SDS-PAGE) analysis of total proteins was used to detect recombinant protein expression. No IPTG and cells transformed with pGEX-4 T-1 or pET30a and induced with IPTG were used as negative controls.

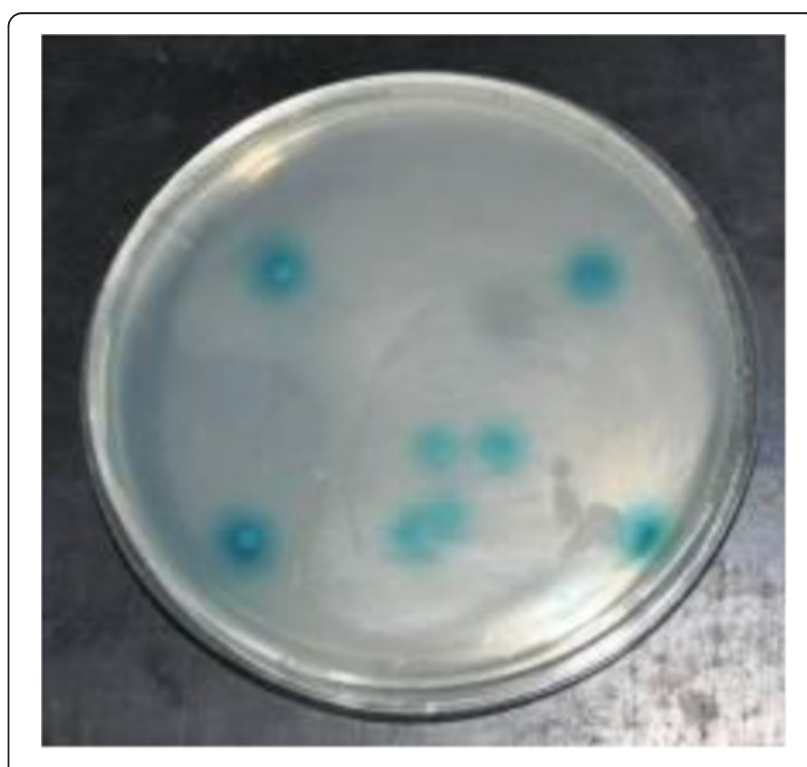

Figure 9 Yeast rotary verify to PoC1q and PoAkirin1. 


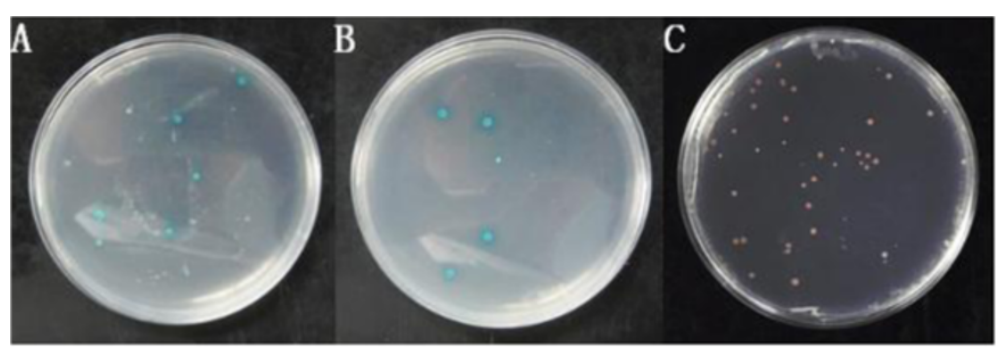

Figure 10 Growth yeast transformed with the PoHEPN deletion segment and PoAkirin1 in SD/-Leu/-Trp/x-a-gal. A: pohepn1; B: pohepn2; C: pohepn3.

\section{Western blotting analysis}

The protein concentrations of the samples were determined using an Enhanced BCA Protein Assay Kit (Beyotime Institute of Biotechnology, China). Recombinant PoHEPN and PoAkirin1 were serially diluted (to $5 \mathrm{ng}$ ) and separated by 12\% SDS-PAGE and transferred to a BioTrace NT Nitrocellulose Transfer Membrane (PALL, USA). The membranes were blocked and then incubated with a $1 \mu \mathrm{g} / \mathrm{mL}$ dilution of the primary antibody (monoclonal antibodies against His or GST (glutathioneS-transferase; Uscn Life Science Inc., Wuhan, China)). After washing, the membranes were incubated with the secondary antibody, horseradish peroxidase-conjugated goat anti-mouse IgG (1:1,000 dilutions) (Beyotime Institute of Biotechnology, China). Reactive proteins were detected using chemiluminescence (ECL Western Blotting Analysis System, Thermo Fisher Scientific Inc., IL, USA).

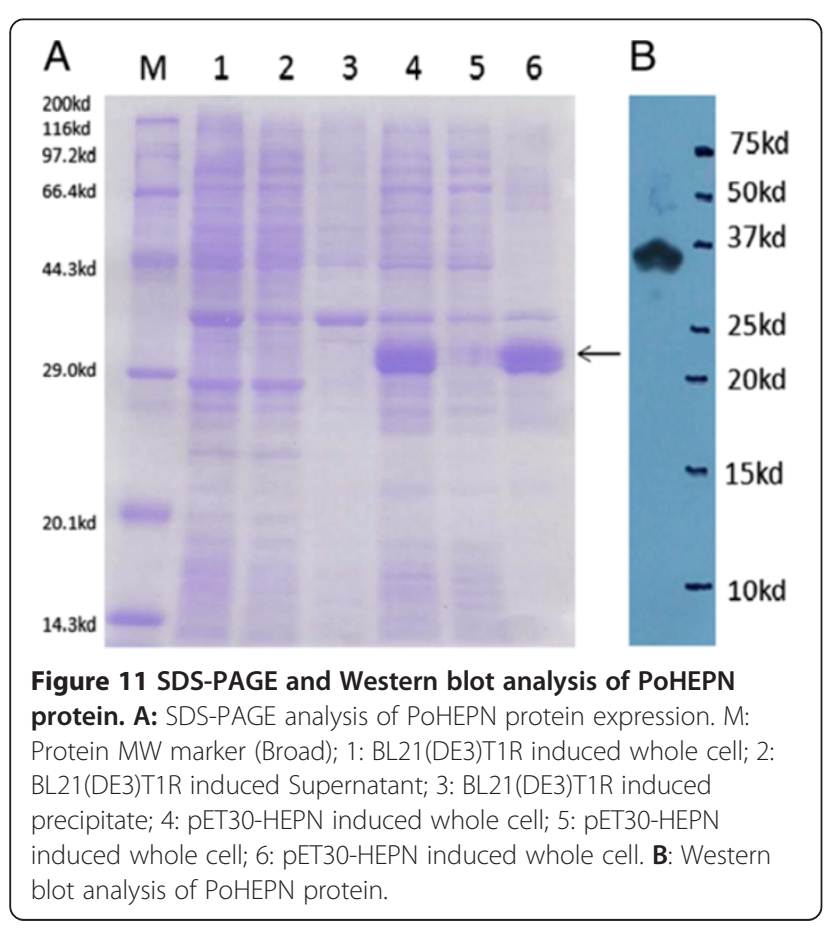

\section{Results}

Cloning and sequence analysis of PoAkirin 1

The PoAkirin1 cDNA acquired from the full-length cDNA Library of Japanese flounder comprised a 5'UTR of $202 \mathrm{bp}$, an ORF of $564 \mathrm{bp}$ encoding a polypeptide of amino acids and a 521-bp 3'UTR with a poly (A) tail (Figure 2). Phylogenetic analysis showed that the PoAkirin1 gene clustered together with fish Akirin1 genes (Figure 3, 4).

The PoAkirin1 cDNA encodes a putative protein of 187 amino acids with a predicted molecular mass of $21 \mathrm{kDa}$ and an isoelectric point (pI) of 9.22. A putative NLS was predicted in the N-terminus of the protein (Figure 2). The sequence of PoAkirin1 has been submitted to GenBank with the accession number KC190111.

\section{Cloning and testing the bait construct for autoactivation} Bait plasmid (100 ng; pGBKT7-PoAkirin1) was transformed into yeast strainY2H Gold and selected on medium SD/Trp (lacking Trp). No autoactivation of fish Akirin1 expression was observed (Figure 5).

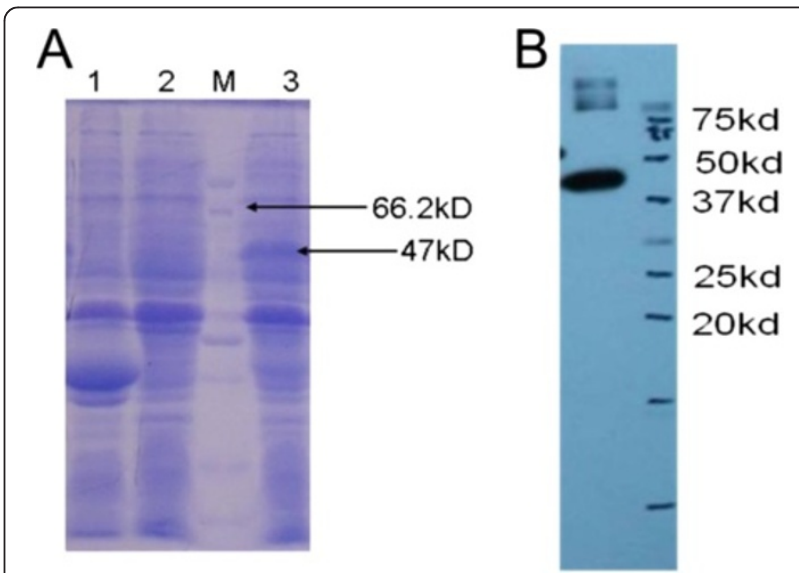

Figure 12 SDS-PAGE and Western blot analysis of PoAkirin1 protein. A: SDS-PAGE analysis of PoAkirin1 protein expression. 1: pGEX4T-1 induced; 2: pGEX4T-1- PoAkirin1 non-induced; 3: pGEX4T1- PoAkirin1 induced; M: Protein MW marker. B: Western blot analysis of PoAkirin1. 


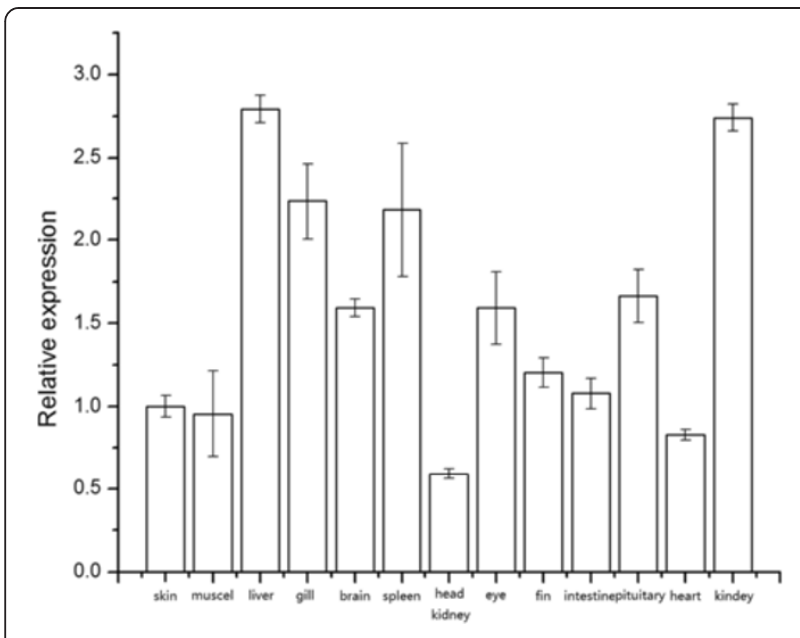

Figure 13 Expression pattern of PoAkirin1 in different tissues of the Japanese flounder detected by quantitative RT-PCR.

PoAkirin 1 mRNA was expressed in all tissues detected. The $\beta$-actin gene was used as an internal control to calibrate the cDNA template for all the samples. All data are expressed as the mean \pm S.D. $(n=3)$.

Yeast two-hybrid screening of a Japanese flounder library using bait pGBKT7-PoAkirin1

Using PoAkirin1 as the bait to screen interactions in the library, 49 positive clones were selected. Yeast plasmids extracted from positive clones were transformed into $E$. coli TOP10 and sequenced. DNAMAN was used to analyze the sequence information and BLASTP at the National Center for Biotechnology Information (http:// ncbi.nlm.nih.gov/blast) was used to perform the alignments. The results showed that there were seven possible interacting proteins (CD63, HEPN, C1q, T-complex protein 1 , voltage-dependent anion channel, asparaginyl endopeptidase and Chaperonin) among the 49 positive clones (Figure 6). To verify the interaction of the

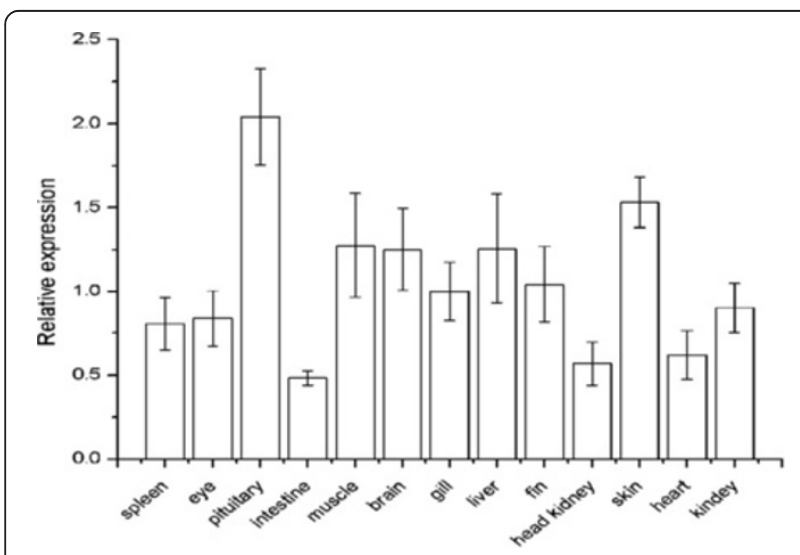

Figure 14 Expression pattern of PoHEPN in different tissues of the Japanese flounder detected by quantitative RT-PCR. POHEPN mRNA was expressed in all tissues. The $\beta$-actin gene was used as an internal control to calibrate the cDNA templates for all samples. Data are expressed as the mean \pm S.D. $(n=3)$.

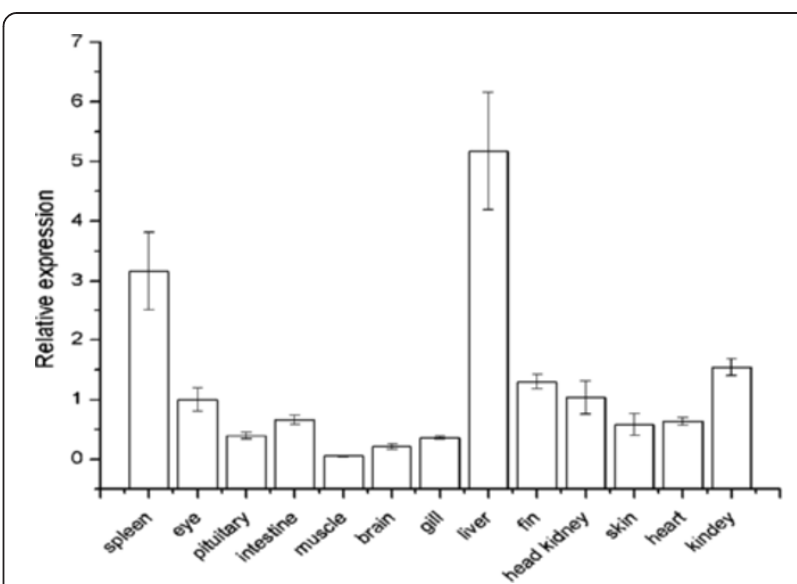

Figure 15 Expression pattern of PoC1q in different tissues of Japanese flounder detected by quantitative RT-PCR. POClq was expressed in all tissues, with higher expression levels in the liver. The $\beta$-actin gene was used as an internal control to calibrate the cDNA templates for all the samples. Data are expressed as the mean \pm S.D. $(n=3)$.

proteins, we chose the possible immune-related protein (C1q) (Figure 7) and the protein predicted in the nucleus (HEPN) (Figure 8) to perform yeast rotary and domain verification tests. The results showed that the yeast grew with a blue color in the yeast rotary verification test using PoC1q and PoAkirin1, and the yeast expressing PoHEPN with a 96-223 aa segment deleted and PoAkirin with a 146-223 aa segment deleted were blue when grown on SD/-Leu/-Trp/x- $\alpha$-gal. (Figures 1, 9, and 10).

\section{PoAkirin 1 and PoHEPN proteins expression in E. coli and western blotting analysis}

The recombinant plasmid pGEX-4T-1-Akirin and empty plasmid pGEX-4T-1 were transformed into E. coli. After induction by $1 \mathrm{mM}$ IPTG for $4 \mathrm{~h}$, bacterial lysates contained a protein band with a $\mathrm{Mr}$ of approximately $47 \mathrm{kDa}$, as anticipated. The lysates from bacteria transformed with the empty vector pGEX-4 T-1 and that had no IPTG induction did not contain this band (Figures 12A).

Similarly, after induction by $1 \mathrm{mM}$ IPTG(Isopropyl $\beta$ D-1-Thiogalactopyranoside) for $4 \mathrm{~h}$, bacterial lysates with the recombinant plasmid pET30a-HEPN showed a protein band with an $\mathrm{Mr}$ of approximately $31 \mathrm{kDa}$, which corresponded to the predicted size. This band was not present in the negative control bacterial lysates (Figures 11A).

The recombinant PoHEPN and PoAkirin1 contain His and GST tags; therefore, western blotting was used to detect the expressed proteins using His and GST monoclonal antibodies (Figures 11B, 12B). 


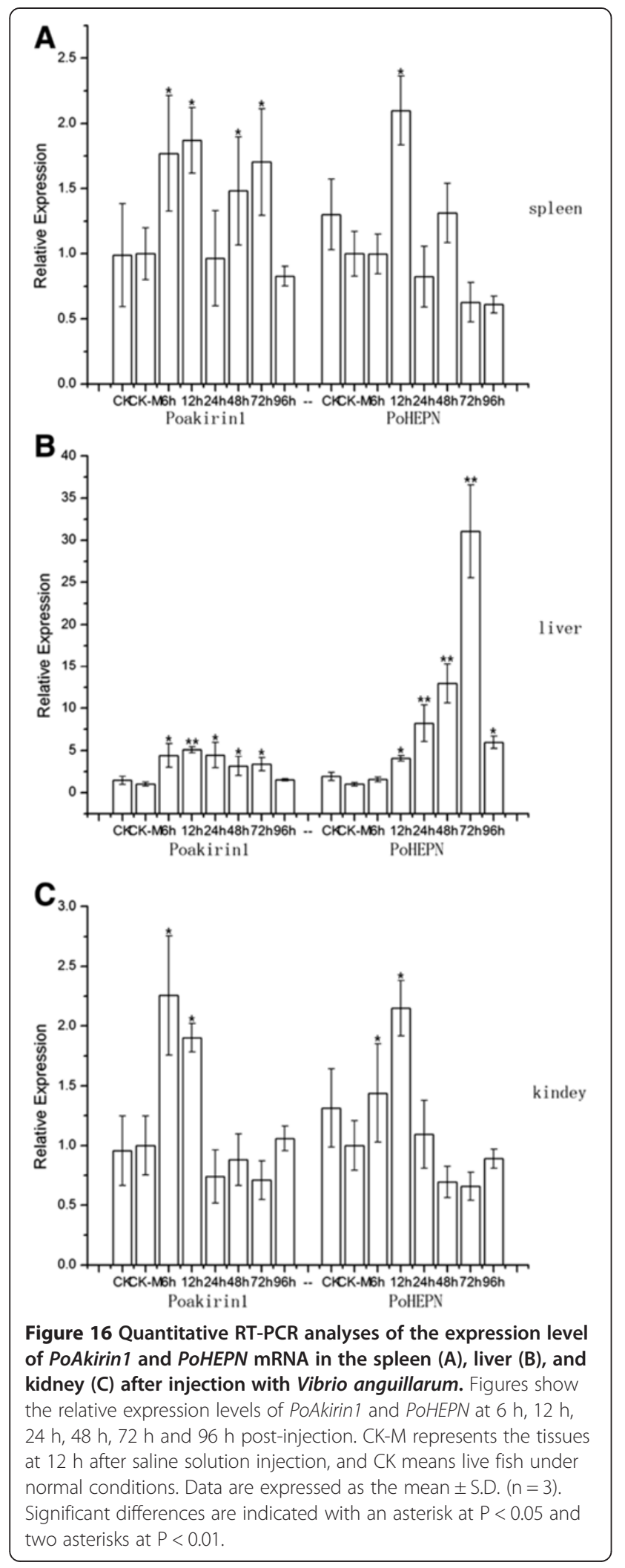

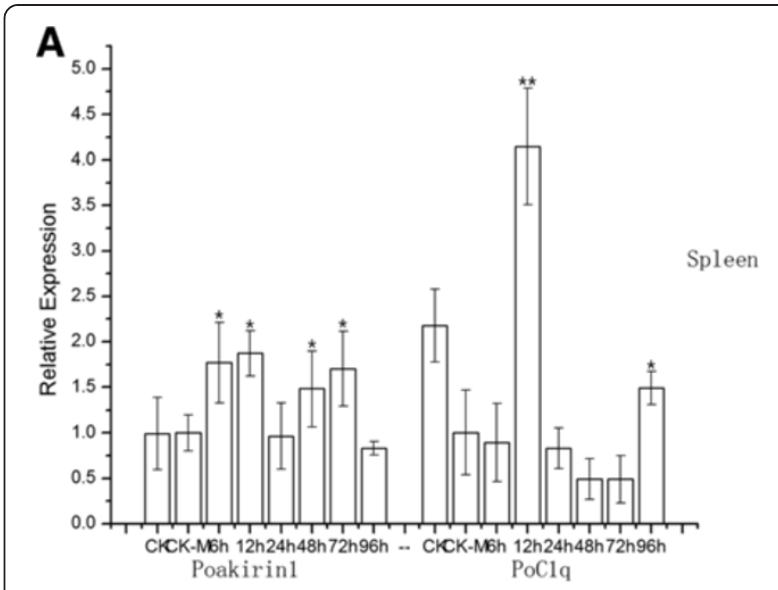
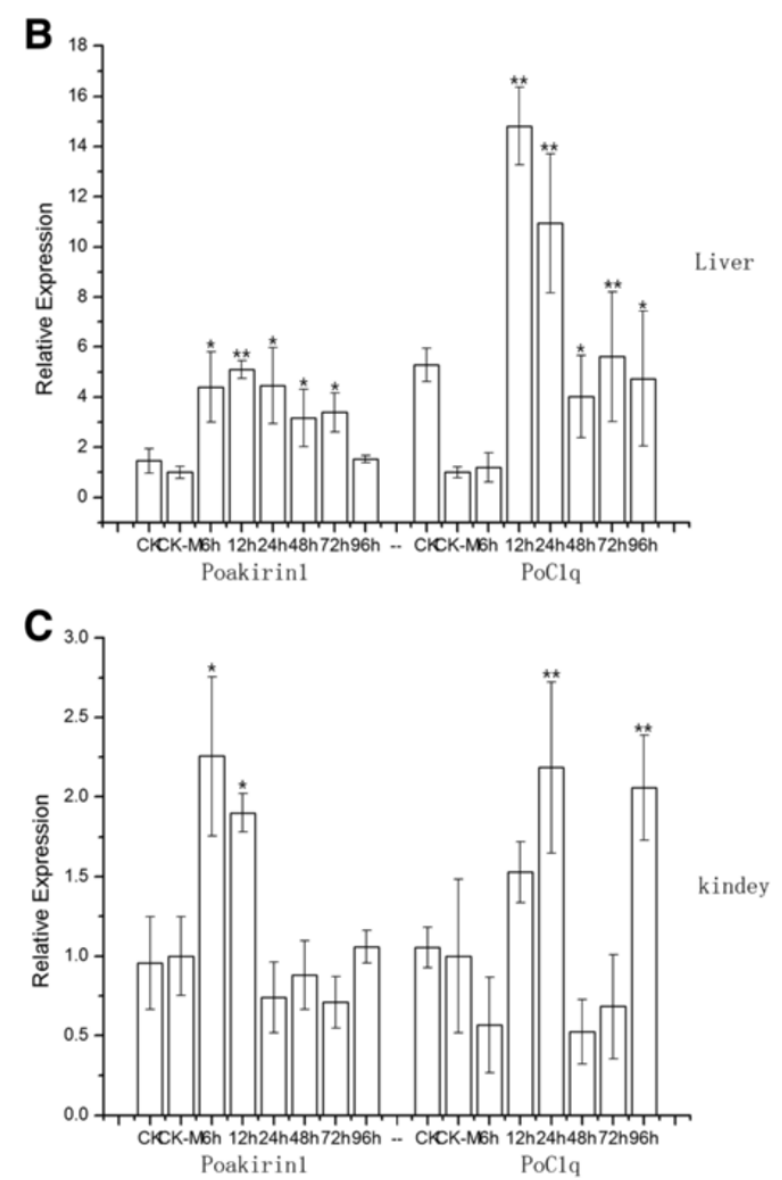

Figure 17 Quantitative RT-PCR analysis of the expression levels of PoAkirin1 and PoC1q mRNA, relative to $\beta$-actin mRNA, in the spleen (A), liver (B), and kidney (C) after injection with Vibrio anguillarum. Graphs show the relative expression levels of PoAkirin 1 and PoCla at $6 \mathrm{~h}, 12 \mathrm{~h}, 24 \mathrm{~h}, 48 \mathrm{~h}, 72 \mathrm{~h}$ and $96 \mathrm{~h}$ post-injection. CK-M represents the tissues at $12 \mathrm{~h}$ after saline solution injection, and CK represents live fish under normal conditions. Data are expressed as the mean \pm S.D. $(n=3)$. Significant differences are indicated with an asterisk at $\mathrm{P}<0.05$ and two asterisks at $\mathrm{P}<0.01$. 


\section{Sequence analysis and expression profile of PoAkirin1, PoHEPN, and Poc1q in various tissues}

Yeast two-hybrid screening identified a 672-bp PoHEPN cDNA, which encoded a protein with a conserved HEPN domain at the C-terminus. The PoC1q gene was $528 \mathrm{bp}$, and analysis of the predicted protein indicated that it contained a conserved $\mathrm{C} 1 \mathrm{q}$ domain at the C-terminus (Figures 7 and 8).

RT-qPCR was used to quantify the expressions of PoAkirin1, PoHEPN and PoClq mRNA in different flounder tissues in normal and pathogen-challenged individuals. The mRNA transcripts of PoAkirin1, PoHEPN and $P o C 1 q$ were detected in all tissues of normal individuals. The PoAkirin1 mRNA showed the highest expression in liver, and was highly expressed in the spleen and kidney. PoAkirin1 was also expressed in the other tissues (Figure 13). In most tissues examined, the POHEPN expression levels did not differ significantly, with slightly higher levels in the pituitary and skin. In comparison with other tissues, PoClq expression was relatively high in the brain, gills, pituitary, skin, heart and eyes. In the liver and spleen, the expression of PoClq significantly higher compared with other tissues $(\mathrm{p}<0.01)$. The expression in the liver was 25 and 100 times higher than in the brain and muscle, respectively. (Figures 14 and 15).

To determine the expression profile of PoAkirin1, PoHEPN and PoC1q mRNA in the challenged flounder, their mRNA levels were examined in three tissues. As shown in Figures 16 and 17, after infection by the bacteria, PoAkirin1 expression initially increased and then decreased in the three tissues (liver, spleen and kidney); the highest expression was detected at 6-24 h. Similar to PoAkirin1, the PoHEPN expression could also be induced by bacteria, but the response time was $6 \mathrm{~h}$ later than that of PoAkirin1 and the significant increment started at 12 h. PoClq expression was induced at 12 and $24 \mathrm{~h}$ by the bacteria, and showed a relatively large increase. In the kidney and spleen, the expression levels of PoC1q were significantly higher than those of the normal control at 24 and $12 \mathrm{~h}$, respectively. Significantly higher levels of $\mathrm{PoCl} q$ were also detected at 12 and $24 \mathrm{~h}$ in the liver (Figures 16 and 17).

\section{Discussion}

\section{The HEPN domain and the sacsin protein}

Spastic ataxia of Charlevoix-Saguenay (SACS) originated from the Lac-Saint-Jean region, Quebec, in Canada. The sacsin gene, the pathogenic gene of SACS, consists of nine exons, including a gigantic exon of over $12.8 \mathrm{kbp}$, which is the biggest exon in vertebrates. The gene encodes the sacsin protein, $65 \%$ certain to be located in the in the nucleus. By SMART [29] analysis, this protein has a recognizable DnaJ conserved domain, which is involved in the interaction of sacsin and Hsp70. The sacsin protein has the other three domains, including a UBQ region at the $\mathrm{N}$-terminus, a HEPN domain at the $\mathrm{C}$-terminus and another DnaJ region upstream of the HEPN domain.

The HEPN domain comprises 110 amino acid residues at $\mathrm{C}$-terminus of the sacsin protein. In some invertebrates, bacteria and archaea, sacsin homologous proteins have this conserved domain.

\section{Proteins with HEPN Domains are of three main types:}

a) A single HEPN domain. In many types of bacterium, the protein is usually followed by nucleotidyltransferase (NT), which is often in a different reading frame, with both ORFs overlapping. Only two exceptions were found: in the genomes of Pyrococcus furiosus and $P$. abyssi, the HEPN protein does not exist after the NT protein.

b) Two HEPN domains, the N-terminal NT domain and C-terminal HEPN domain.

c) HEPN proteins with a variety of conserved domains. These occur mainly in fish and mammals (monkeys, rats, mice and humans). The HEPN domain is located at the $\mathrm{C}$ terminus of the protein, adjacent to the DnaJ domain. However, conserved HEPN domains in lower eukaryotes have not been found [30].

The function of the HEPN domain in sacsin is not clear and is difficult to predict. HEPN has an important role in stabilizing nucleotide binding in complexes formed with the DnaJ domain and may be involved in determining the specificity. In diseases caused by sacsin mutations, sacsin mutant proteins have an incomplete DnaJ and HEPN conserved domain [31]. Thus, the functions of HEPN and sacsin proteins require further study.

\section{C1q}

Complement component $\mathrm{C} 1$ is composed of three subunits: $\mathrm{Clq}, \mathrm{C} 1 \mathrm{r}$ and $\mathrm{C} 1 \mathrm{~s}$ [32]. C1q, in C1, combined with immune complexes (IC) or other non-immune complexes, activates the classical pathway of complement activation. The $\mathrm{C} 1 \mathrm{q}$ complement system, activated via the classic, bypass and mannose-binding lectin activation pathways, is composed of 30 types of protein. $\mathrm{C} 1 \mathrm{q}$ has two functional units: a collagen-like region (CLR) near the $\mathrm{N}$-terminus and a globular region (GR) at C-terminus. The CLR of C1q, with C1r and C1s, forms the C1 macromolecules $(\mathrm{C} 1 \mathrm{qC} 1 \mathrm{r} 2 \mathrm{C} 1 \mathrm{~s} 2)$. After the recognizing and binding to the GR through the $\mathrm{IC}, \mathrm{C} 1 \mathrm{r}$ and $\mathrm{C} 1 \mathrm{~s}$ are activated, thereby initiating the classical pathway. When complement is activated, a number of complement pro- 
teins can be cleaved into a variety of small cell surface fragments that are recognized by complement receptors. The primary function of the cell surface complement receptors is to promote the natural immune system to remove the foreign proteins, cell debris and microorganisms from the circulatory system $[33,34]$.

\section{PoAkirin1 interacts with the $\mathrm{N}$-terminus of PoHEPN}

The HEPN conserved domain is predicted to locate in the 97-223 amino acids region of PoHEPN. Therefore, in the mutation experiment of PoHEPN segment deletion, we constructed three vectors comprising 1-96aa (pohepn1), 1-146aa (pohepn2), and 147-224aa (pohepn3), to verify whether PoHEPN can interact with PoAkirin1, where the binding site of PoHEPN with PoAkirin1 is, whether the interaction is associated with the conserved sequences, and whether the incomplete sequence of PoHEPN retains its binding activity. The result showed that the region of PoHEPN that binds with PoAkirin1 is located in the 1-96aa region, before the HEPN domain, and is unrelated with the HEPN domain. This suggests that the nucleoprotein PoAkirin1 has no transcriptional activation activity and must be combined with other proteins (transcription factors or other DNA-binding proteins), such as PoHEPN, which can bind DNA and initiate expression of immunerelated genes as a complex. In this process, PoAkirin1 is likely to play a specific-enhancer role; however, this hypothesis should be tested by further experimentation.

\section{PoAkirin 1 and PoHEPN expression in E. coli and western blotting analysis}

Prokaryotic fusion protein expression vectors for PoAkirin1 and PoHEPN were constructed and the recombinant PoHEPN and PoAkirin1 were successfully expressed in E. coli. The recombinant proteins were verified by western blotting. The recombinant proteins will be separated and purified. These recombinant proteins will be useful for further investigation of the function of PoAkirin1, such as to verify the binding activity of PoAkirin1 with PoHEPN, changes in protein expression levels, in situ hybridization, crystal structure, and protein activity.

\section{Expression profiles of PoAkirin1, PoHEPN, PoC1q}

To investigate the expression profile of PoAkirin1, the mRNA expressions of PoHEPN and PoC1q in different tissues from normal and $V$. anguillarum challenged fish were examined at different time points in three important immune organs: the kidney, spleen and liver. The results showed that PoAkirin1 was highly expressed in all the three tissues. The expression profile suggested that PoAkirin1 might be involved in growth, development and, especially, immunity. In most normal tissues, the expression of PoHEPN did not differ significantly. It was only slightly higher in the pituitary and skin than in other tissues. PoC1q was hardly expressed in muscle, slightly expressed in brain, gills, pituitary, skin, heart and eyes, but highly expressed in the liver, spleen, kidney and head kidney. In the liver and spleen, the expression level is significantly different compared with other tissues $(\mathrm{p}<0.01)$. The expression in liver is 25 times higher than in the brain and 100 times higher than in the muscle. The reason for this tissue-specific expression may relate to their different functions. The sacsin protein has mainly been associated with nerve function [31,35]. Therefore, although it is expressed in various tissues and organs, its expression is higher in the pituitary and the skin. However, for the $\mathrm{C} 1 \mathrm{q}$ protein, as a subunit of complement $\mathrm{C} 1$, its distribution is mainly in the immune tissues, where it is involved in a series of signal transductions.

The expressions of the PoAkirin1, PoHEPN, and PoC1q gene were all induced by bacterial challenge, where the expression of PoAkirin1 reached its highest point reached after 12-24 h. Interestingly, however, the responses to the bacterial challenge of PoHEPN and PoC1q were slower than that of PoAkirin1, which may indicate that PoHEPN and PoC1q are located downstream in the response to bacterial challenge, and that the change in expression of PoAkirin1 leads directly to changes in the expressions of PoHEPN and PoC1q. However, the specific relationship between the upstream and downstream proteins in this signaling pathway requires further study.

\section{Conclusions}

In this paper, we cloned the Akirin1 homologous gene, PoAkirin1, from Japanese flounder and identified two proteins that potentially interact with PoAkirin1. The expression patterns of PoAkirin1 and the genes encoding the interacting proteins are closely associated with immunity. Bacteria can induce the expression of these genes; therefore, the function of this protein merits further investigation, particularly in the context of protecting fish populations.

\section{Competing interests}

The authors declare that they have no competing interests.

\section{Authors' contributions}

S-LC obtained the project. S-LC and C-GY designed the study. C-GY, X-LW, BZ, BS, and S-SL carried out the molecular genetic studies, participated in the sequence alignment and drafted the manuscript. C-GY, X-LW, and BZ carried out the immunoassays. BS and S-SL participated in the statistical analysis. S-LC revised the manuscript. All authors read and approved the final manuscript.

\section{Acknowledgments}

This work was supported by grants from the State 863 High-Technology R\&D Project of China (2012AA092203, 2012AA10A408), the 973 National Basic Research Program of China (2010CB126303), and the Taishan Scholar Project Fund of Shandong, China. 


\section{Author details}

${ }^{1}$ Yellow Sea Fisheries Research Institute, Chinese Academy of Fisheries Sciences, Qingdao 266071, China. ${ }^{2}$ Yangtze River Fisheries Research Institute, Chinese Academy of Fishery Sciences, Wuhan 430223, China. ${ }^{3}$ Translational Center for Stem Cell Research, Tongji Hospital, Stem Cell Research Center, Tongji University School of Medicine, Shanghai 200065, China. ${ }^{4}$ Bohai Sea Fisheries Research Institute of Tianjin, Tianjin, China.

Received: 27 February 2012 Accepted: 22 April 2013

Published: 7 May 2013

\section{References}

1. Auerbach $\mathrm{D}$, Thaminy S, Hottiger MO, Stagljar I: The post-genomic era of interactive proteomics: facts and perspectives. Proteomics 2002, 2(6):611-623.

2. Ito T, Tashiro K, Muta S, Ozawa R, Chiba T, Nishizawa M, Yamamoto K, Kuhara S, Sakaki Y: Toward a protein-protein interaction map of the budding yeast: A comprehensive system to examine two-hybrid interactions in all possible combinations between the yeast proteins. Proc Natl Acad Sci USA 2000, 97(3):1143-1147.

3. Rain JC, Selig L, De Reuse H, Battaglia V, Reverdy C, Simon S, Lenzen G, Petel F, Wojcik J, Schachter $V$, et al: The protein-protein interaction map of Helicobacter pylori. Nature 2001, 409(6817):211-215.

4. Terradot L, Durnell N, Li M, Ory J, Labigne A, Legrain P, Colland F, Waksman $\mathrm{G}$ : Biochemical characterization of protein complexes from the Helicobacter pylori protein interaction map: strategies for complex formation and evidence for novel interactions within type IV secretion systems. Mol Cell Proteomics 2004, 3(8):809-819.

5. Giot L, Bader JS, Brouwer C, Chaudhuri A, Kuang B, Li Y, Hao YL, Ooi CE, Godwin B, Vitols $E$, et al: A protein interaction map of Drosophila melanogaster. Science 2003, 302(5651):1727-1736.

6. Ito T, Chiba T, Ozawa R, Yoshida M, Hattori M, Sakaki Y: A comprehensive two-hybrid analysis to explore the yeast protein interactome. Proc Natl Acad Sci USA 2001, 98(8):4569-4574.

7. Li S, Armstrong CM, Bertin N, Ge H, Milstein S, Boxem M, Vidalain PO, Han $J \mathrm{D}$, Chesneau A, Hao T, et al: A map of the interactome network of the metazoan C. elegans. Science 2004, 303(5657):540-543.

8. Goto A, Matsushita K, Gesellchen V, El Chamy L, Kuttenkeuler D, Takeuchi O, Hoffmann JA, Akira S, Boutros M, Reichhart JM: Akirins are highly conserved nuclear proteins required for NF-kappaB-dependent gene expression in drosophila and mice. Nat Immunol 2008, 9(1):97-104.

9. de la Fuente J, Almazan C, Blas-Machado U, Naranjo V, Mangold AJ, Blouin EF, Gortazar C, Kocan KM: The tick protective antigen, 4D8, is a conserved protein involved in modulation of tick blood ingestion and reproduction. Vaccine 2006, 24(19):4082-4095.

10. Almazan C, Blas-Machado U, Kocan KM, Yoshioka JH, Blouin EF, Mangold AJ, de la Fuente J: Characterization of three Ixodes scapularis CDNAs protective against tick infestations. Vaccine 2005, 23(35):4403-4416.

11. Marshall A, Salerno MS, Thomas M, Davies T, Berry C, Dyer K, Bracegirdle J, Watson T, Dziadek M, Kambadur R, et al: Mighty is a novel promyogenic factor in skeletal myogenesis. Exp Cell Res 2008, 314(5):1013-1029.

12. Komiya Y, Kurabe N, Katagiri K, Ogawa M, Sugiyama A, Kawasaki Y, Tashiro F: A novel binding factor of 14-3-3beta functions as a transcriptional repressor and promotes anchorage-independent growth, tumorigenicity, and metastasis. J Biol Chem 2008, 283(27):18753-18764.

13. Macqueen DJ, Kristjansson BK, Johnston IA: Salmonid genomes have a remarkably expanded akirin family, coexpressed with genes from conserved pathways governing skeletal muscle growth and catabolism. Physiol Genomics 2010, 42(1):134-148.

14. Macqueen DJ, Johnston IA: Evolution of the multifaceted eukaryotic akirin gene family. BMC Evol Biol 2009, 9:34.

15. Yang CG, Wang XL, Wang L, Zhang B, Chen SL: A new Akirin1 gene in turbot (Scophthalmus maximus): molecular cloning, characterization and expression analysis in response to bacterial and viral immunological challenge. Fish Shellfish Immunol 2011, 30(4-5):1031-1041.

16. Nam BH, Hirono I, Aoki T: Bulk isolation of immune response-related genes by expressed sequenced tags of Japanese flounder Paralichthys olivaceus leucocytes stimulated with Con A/PMA. Fish Shellfish Immunol 2003, 14(5):467-476.

17. Nam BH, Yamamoto E, Hirono I, Aoki T: A survey of expressed genes in the leukocytes of Japanese flounder, Paralichthys olivaceus, infected with Hirame rhabdovirus. Dev Comp Immunol 2000, 24(1):13-24.
18. Park EM, Kang JH, Seo JS, Kim G, Chung J, Choi TJ: Molecular cloning and expression analysis of the STAT1 gene from olive flounder. Paralichthys olivaceus. BMC Immunol 2008, 9:31

19. Chen SL, Wang ZJ, Xu MY, Gui JF: Molecular identification and expression analysis of natural resistance associated macrophage protein (Nramp) cDNA from Japanese flounder (Paralichthys olivaceus). Fish Shellfish Immunol 2006, 20(3):365-373.

20. Xu TJ, Chen SL, Ji XS, Tian YS: MHC polymorphism and disease resistance to Vibrio anguillarum in 12 selective Japanese flounder (Paralichthys olivaceus) families. Fish Shellfish Immunol 2008, 25(3):213-221.

21. Zhang YX, Chen SL: Molecular identification, polymorphism, and expression analysis of major histocompatibility complex class IIA and B genes of turbot (Scophthalmus maximus). Mar Biotechnol (NY) 2006, 8(6):611-623.

22. Chen S, Xu M, Ji X, Yu G: Cloning and characterization of natural resistance associated macrophage protein (Nramp) cDNA from red sea bream (Chrysophrys major). Fish Shellfish Immunol 2004, 17:305-313.

23. Saitou N, Nei M: The neighbor-joining method: a new method for reconstructing phylogenetic trees. Mol Biol Evol 1987, 4(4):406-425.

24. Tamura K, Dudley J, Nei M, Kumar S: MEGA4: Molecular Evolutionary Genetics Analysis (MEGA) software version 4.0. Mol Biol Evol 2007, 24(8): 1596-1599.

25. Bustin SA, Benes V, Garson JA, Hellemans J, Huggett J, Kubista M, Mueller R, Nolan T, Pfaffl MW, Shipley GL, et al: The MIQE guidelines: minimum information for publication of quantitative real-time PCR experiments. Clin Chem 2009, 55(4):611-622.

26. Zheng WJ, Sun L: Evaluation of housekeeping genes as references for quantitative real time RT-PCR analysis of gene expression in Japanese flounder (Paralichthys olivaceus). Fish Shellfish Immunol 2011, 30(2):638-645.

27. Martin-Antonio B, Jimenez-Cantizano RM, Salas-Leiton E, Infante C, Manchado M: Genomic characterization and gene expression analysis of four hepcidin genes in the redbanded seabream (Pagrus auriga). Fish Shellfish Immunol 2009, 26(3):483-491.

28. Kurobe T, Yasuike M, Kimura T, Hirono I, Aoki T: Expression profiling of immune-related genes from Japanese flounder Paralichthys olivaceus kidney cells using cDNA microarrays. Dev Comp Immunol 2005, 29(6):515-523

29. Letunic I, Goodstadt L, Dickens NJ, Doerks T, Schultz J, Mott R, Ciccarelli F, Copley RR, Ponting CP, Bork P: Recent improvements to the SMART domain-based sequence annotation resource. Nucleic Acids Res 2002, 30(1):242-244

30. Grynberg M, Erlandsen H, Godzik A: HEPN: a common domain in bacterial drug resistance and human neurodegenerative proteins. Trends Biochem Sci 2003, 28(5):224-226.

31. Engert JC, Berube P, Mercier J, Dore C, Lepage P, Ge B, Bouchard JP, Mathieu J, Melancon SB, Schalling M, et al: ARSACS, a spastic ataxia common in northeastern Quebec, is caused by mutations in a new gene encoding an 11.5-kb ORF. Nat Genet 2000, 24(2):120-125.

32. Lepow IH, Naff GB, Todd EW, Pensky J, Hinz CF: Chromatographic resolution of the first component of human complement into three activities. J Exp Med 1963, 117:983-1008.

33. Kishore U, Leigh LE, Eggleton $P$, Strong P, Perdikoulis MV, Willis AC, Reid KB: Functional characterization of a recombinant form of the C-terminal, globular head region of the B-chain of human serum complement protein, C1q. Biochem J 1998, 333(Pt 1):27-32.

34. Lu J, Wu X, Teh BK: The regulatory roles of C1q. Immunobiology 2007 212(4-5):245-252.

35. Bouchard JP, Barbeau A, Bouchard R, Bouchard RW: Autosomal recessive spastic ataxia of Charlevoix-Saguenay. Can J Neurol Sci 1978, 5(1):61-69.

\section{doi:10.1186/1471-2199-14-10}

Cite this article as: Yang et al:: Screening and analysis of PoAkirin 1 and two related genes in response to immunological stimulants in the Japanese flounder (Paralichthys olivaceus). BMC Molecular Biology 2013 14:10. 